\title{
BMJ Open Quality Improving hepatitis C screening and diagnosis in patients born between 1945 and 1965 in a safety-net primary care clinic
}

\author{
Smita Bakhai, ${ }^{1}$ Naren Nallapeta, ${ }^{1}$ Mohammad El-Atoum, ${ }^{1}$ Tenzin Arya, ${ }^{1}$ \\ Jessica L Reynolds ${ }^{2}$
}

To cite: Bakhai S, Nallapeta N, El-Atoum M, et al. Improving hepatitis $C$ screening and diagnosis in patients born between 1945 and 1965 in a safety-net primary care clinic. BMJ Open Quality 2019;8:e000577. doi:10.1136/ bmjoq-2018-000577

Received 7 November 2018 Revised 6 August 2019 Accepted 7 September 2019
Check for updates

(c) Author(s) (or their employer(s)) 2019. Re-use permitted under CC BY-NC. No commercial re-use. See rights and permissions. Published by BMJ.

${ }^{1}$ Department of Internal Medicine, University at Buffalo - The State University of New York, Buffalo, New York, USA

${ }^{2}$ Department of Medicine, University at Buffalo - The State University of New York, Buffalo, New York, USA

Correspondence to Dr Smita Bakhai; sybakhai@buffalo.edu

\section{ABSTRACT}

Individuals born between 1945-1965 represent $81 \%$ of all persons chronically infected with hepatitis $\mathrm{C}$ virus (HCV) in the USA and are largely unaware of their positive status. The baseline HCV screening rate in this population in an academic internal medicine clinic at a US hospital was less than $3.0 \%$. The goal was to increase the rate of HCV screening in patients born between 1945 and 1965 to $20 \%$ within 24 months. The quality improvement team used the Plan Do Study Act Model. Outcome measures included HCV antibody screening, HCV RNA positive rate and linkage to hepatology care. Process measures included HCV antibody order and completion rates. The quality improvement team performed a root cause analysis and identified barriers for HCV screening and linkage to care. The key elements of interventions included redesigning nursing workflow, use of health information technology and educating patients, physicians and nursing staff about HCV. The HCV screening rate was $30.3 \%(391 / 1291)$ within 24 months. The HCV antibody positive rate was $43.5 \%$ (170/391), and HCV RNA positive rate was $95.3 \%$ (162/170). HCV infection was diagnosed in $12.5 \%$ (162/1291) of patients or $41.4 \%$ $(162 / 391)$ of the screened population. Of those positive, $70 \%$ (114/162) were linked to hepatology care within the 24-month project timeframe. Eighty percent of patients seen by a hepatologist were treated with direct-acting antivirals agents. The HCV screening rate was sustained at $25.4 \%$ during the post-project 1 -year period. Engagement of a multidisciplinary team and education to patients, physicians and nursing staff were the key drivers for success.

\section{INTRODUCTION}

\section{Quality problem}

An estimated 3.5 million people in the USA are infected with hepatitis $\mathrm{C}$ virus $(\mathrm{HCV})^{1-4}$ with approximately $50 \%$ being unaware of their HCV status. ${ }^{56}$ About $15 \%-25 \%$ of people infected with HCV spontaneously clear the virus, while $75 \%-85 \%$ of individuals develop a chronic infection. ${ }^{2} 78$ Of these chronically infected patients, about $5 \%-20 \%$ will develop liver cirrhosis within 20 years, which is strongly associated with the development of hepatocellular carcinoma. ${ }^{9-11}$ Patients with chronic HCV have higher direct healthcare cost burden, which increases with disease severity. ${ }^{12} 13$

Individuals born between 1945 and 1965 have a sixfold higher prevalence than adults of other ages and represent $81 \%$ of all persons chronically infected with $\mathrm{HCV}^{4}{ }^{14}$ These individuals were likely infected during the 1960s through the 1980s due to lack of universal precautions and infection control procedures. ${ }^{3}{ }^{15}$ Additonally, prior to 1992 , a source of infection was through contaminated blood and blood products before widespread screening of the blood supply was implemented. ${ }^{31516}$ Due to the high prevalence of HCV in individuals born between 1945 and 1965, the US Preventive Services Task Force and the Centers for Disease Control (CDC) have endorsed a one-time screening of those born between 1945 and 1965 for $\mathrm{HCV}^{17-19}$; however, a large percentage of this population have not yet been screened. ${ }^{1620-22}$ Studies suggest that HCV screening through an established primary care provider has a higher likelihood of minimising the gaps in HCV treatment and improve health outcomes for patients. ${ }^{23-28}$ The New York state HCV testing law requires healthcare providers to offer HCV antibody screening to all individuals born between 1945 and 1965 for HCV who are receiving services in primary care settings or inpatient hospital settings. ${ }^{29} 30$ Furthermore, if the individual agrees to be screened and tests positive, the provider must offer follow-up healthcare including a confirmatory HCV RNA test. ${ }^{30}$

Current HCV treatment regimens have revolutionised the HCV therapeutic paradigm. Treatment consists of direct-acting antivirals agents that result in a shorter duration of treatment, minimal side effects, low pill burden and sustained viral response in $90 \%-98 \%$ of patients after 8-12 weeks of 
therapy. ${ }^{831} 32$ With the increased likelihood of achieving sustained viral response due to direct-acting antivirals agents, identifying HCV-positive patients and linking them to providers for treatment is necessary for elimination of HCV. In an academic, safety-net internal medicine clinic in which $70 \%$ of active patients were born between 1945 and 1965, we identified a gap in HCV screening in this population. The aim of this quality improvement project was implemented to increase HCV screening and diagnosis rates in patients born between 1945 and 1965 to $20 \%$ from the baseline rate of less than $3 \%$ within 24 months. Our goal was to improve linkage to hepatology care to $50 \%$ from the baseline rate of $10 \%$ in HCV patients.

\section{METHODS}

\section{Setting}

The internal medicine clinic care team consisted of five attending physicians, 35-40 residents (Academic Internal Medicine Residency Program), nurses, a social worker and administrative staff. The population consisted of underserved and urban patients, and the majority were African-Americans. We created an electronic health record database for this quality improvement project. Patients born between 1945 and 1965, regardless of the presence of any traditional risk factors, that had no prior HCV testing or diagnosis documented in the database were included. A retrospective review of the database revealed a baseline rate for HCV screening of less than $3.0 \%$ in eligible active (seen at least once within 18 months) patients born between 1945 and 1965.

\section{Measurements}

Outcome measures included an increase in HCV antibody screening rates to $20 \%$ in eligible patients, the HCV RNA positive rate and linkage to hepatology care to $50 \%$ from the baseline rate of $10 \%$ in HCV patients. Linkage to care was defined as adherence to the first hepatologist appointment. The process measures included HCV antibody order rate, HCV completion rate, referrals to a hepatology clinic in HCV RNA-positive patients and improvement in physician knowledge in HCV infection. The balance measures, used to determine if improvement in one area is negatively impacting another area, ${ }^{33}$ included increase in patient wait time in the clinic and the cost of treatment. Low improvement targets for the outcome and process measures were set due to the following reasons: (A) insufficient time (20-30 min per visit) to address HCV screening in every eligible patients due to patients presenting with multiple comorbidities, (B) multiple barriers to acceptance of HCV screening in the internal medicine clinic population, $(\mathrm{C})$ lack of an automated electronic health record decision support tool to identify eligible patients including those high risk for substance use disorder and lack of automated HCV test order entry and (D) limited access to a hepatologist.

\section{Implementation \\ Design}

The Plan Do Study Act (PDSA) model of healthcare improvement was used. ${ }^{34}$ The quality improvement team consisted of the internal medicine clinic care team, information technology staff and hepatology clinic staff. The quality improvement team performed a root cause analysis and identified the materials/methods, physician and patient-related barriers for HCV screening and linkage

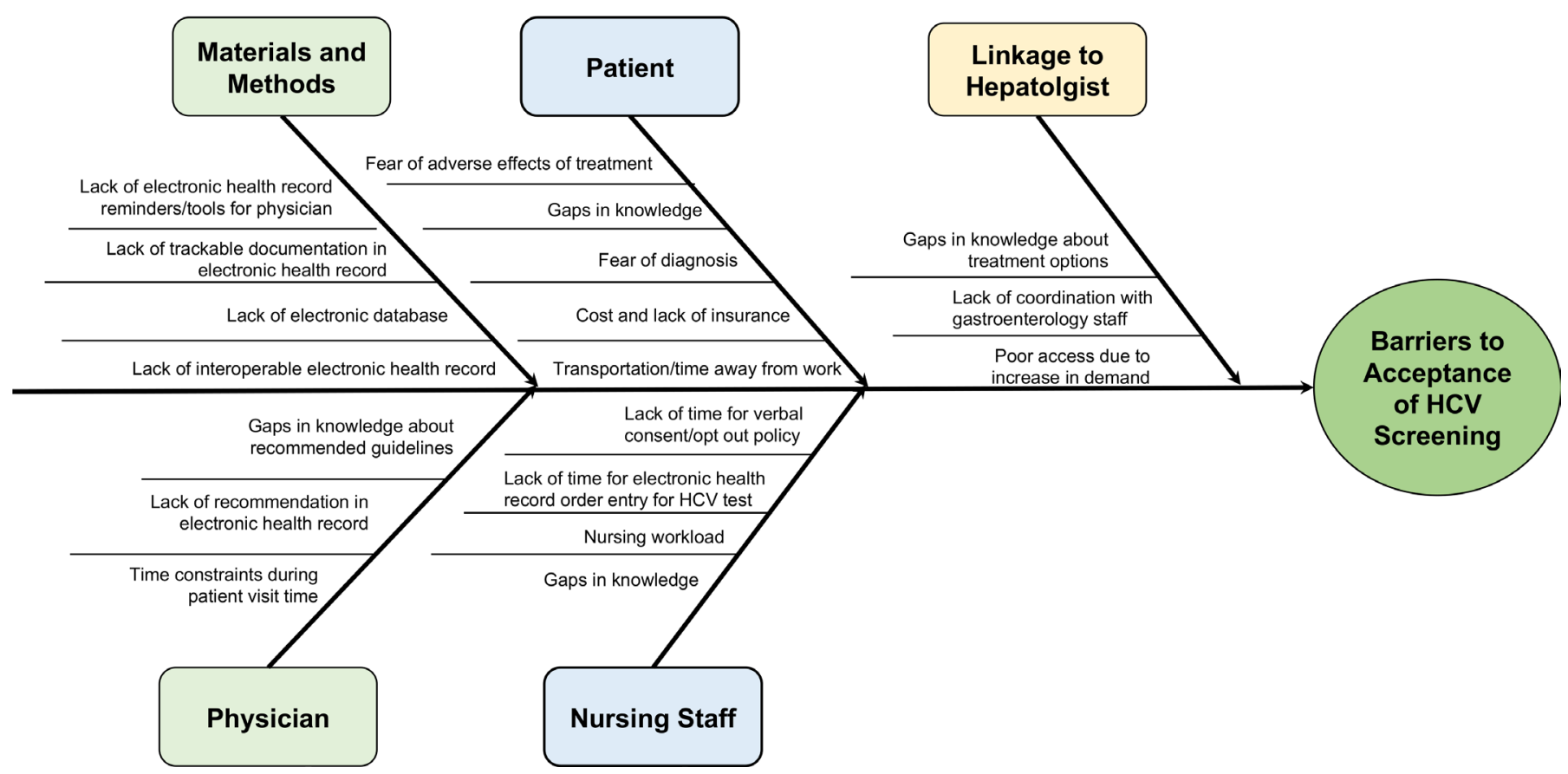

Figure 1 Fishbone diaphragm: root cause analysis barriers to acceptance of HCV screening. HCV, hepatitis C virus. 
Secondary Drivers

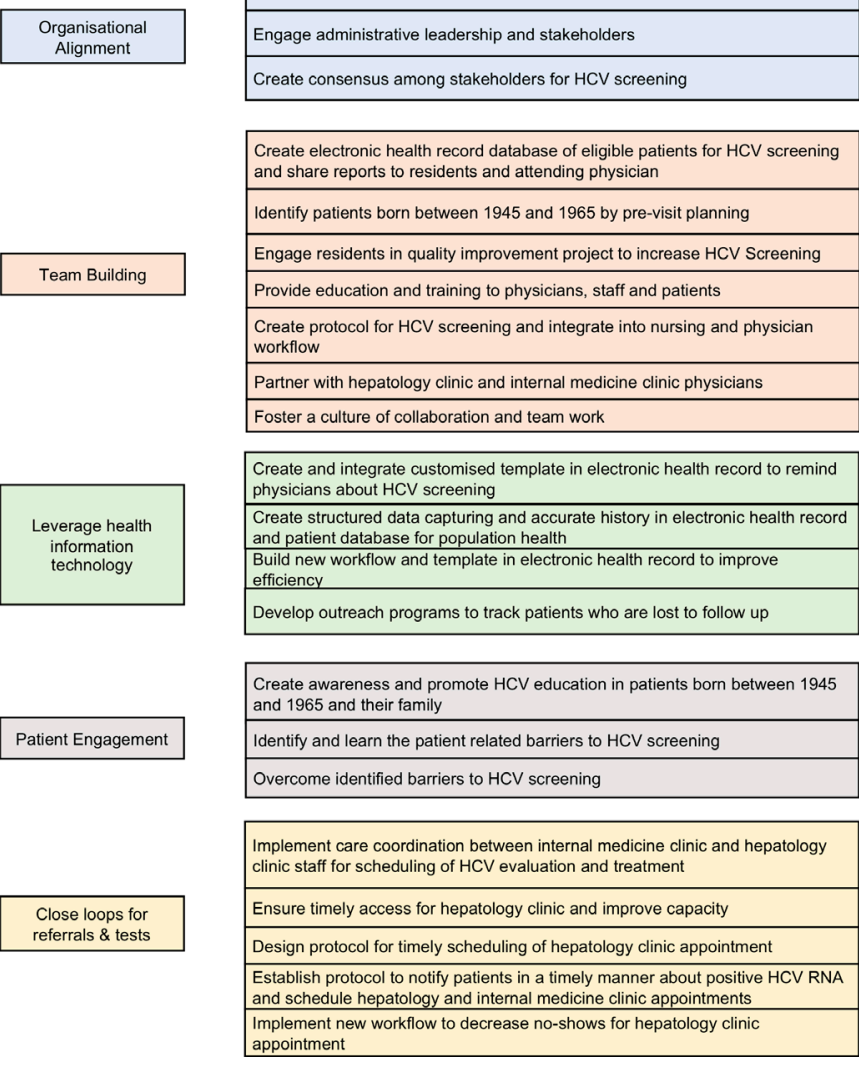

Figure $2 \mathrm{HCV}$ screening drivers diagram. HCV, hepatitis $\mathrm{C}$ virus.

to hepatology care (figure 1). The quality improvement team identified primary and secondary drivers, outlined potential change ideas and developed a driver diagram (figure 2). We identified strengths and prioritised change ideas to overcome the challenges to improve screening rates (table 1 ). We created a process flow map to improve HCV screening (figure 3). We used the SQUIRE 2.0 guidelines for reporting this quality improvement project. ${ }^{35}$ Eligible patients were screened using an HCV antibody test. The laboratory automatically performed a HCV RNA PCR test from the original blood sample (reflex testing), after a positive HCV antibody test. Once a diagnosis of HCV was confirmed, the physician referred the patients to a hepatology clinic for further evaluation and management.

\section{Strategy}

PDSA cycle 1 (June-July 2015): creation of a customised workflow in electronic health record

In June-July of 2015, a customised electronic health record template was created in collaboration with the information technology department to integrate the HCV screening tool. There was no automated electronic health record alert to remind physicians to perform HCV screening in patients born between 1945 and 1965; therefore, a new workflow was created that included offering of HCV screening to the patient by the nursing staff prior to physician evaluation. This offering was fully integrated in the 'routine' nursing workflow prior to the vitals section (i.e. blood pressure, pulse) in the electronic health record. This new workflow served as a reminder to the physicians to use the HCV screening tool and to improve physician's efficiency in ordering of HCV antibody testing.

\section{PDSA cycle 2 (August-September 2015): physician education} about HCV and electronic health record workflow

In August 2015, gaps in the residents' knowledge on HCV were identified by pretest prior to the training. Physicians were educated on HCV infection by a PowerPoint presentation with a small group discussion. Pretest and post-tests were performed, using seven multiple choice questions generated from HCV screening and treatment practice guidelines from the CDC and Infectious Disease Society of America. The physician champion and resident team leader created this assessment test. Physicians were also educated about electronic health record workflow for HCV screening.

\section{PDSA cycle 3 (October-December 2015): nursing education on HCV screening and electronic health record workflow}

The nursing staff was educated about HCV screening in pateints born between 1945 and 1965 and a customised electronic health record workflow. During the nursing assessment, the nursing staff offered HCV screening to patients and documented the patient's verbal consent in the electronic health record prior to the patient and physician interaction. Physicians ordered HCV antibody testing, once verbal consent was obtained. Physicians also provided education to the patient about HCV.

\section{PDSA Cycle 4 (January-March 2016): physician reminders with HCV screening posters}

The team placed the posters in the physician's task boxes to remind them to screen for $\mathrm{HCV}$ in patients born between 1945 and 1965. The physician champion and resident team leaders performed continuous education on HCV screening for the internal medicine residents. The quality improvement team and residents received feedback every 5 weeks by sharing progress reports on the data.

\section{PDSA cycle 5 (April-June 2016): patient education}

The patient education posters were placed in the examination room and in patient discharge areas in the clinic. The team also provided brochures to the patients about HCV testing and possible complications of untreated chronic HCV infection.

PDSA cycle 6 (July-September 2016): physician refresher training about HCV and electronic health record workflow

The physician champion conducted refresher training and education to new interns and other internal medicine clinic physicians about chronic HCV and electronic health record workflow for HCV screening and documentation. 
Table 1 Change ideas tested by the internal medicine clinic to improve HCV screening and diagnosis

\begin{tabular}{|c|c|}
\hline Drivers & Change ideas \\
\hline $\begin{array}{l}\text { Organisational } \\
\text { alignment }\end{array}$ & $\begin{array}{l}\text { Hospital leadership support the Center for Disease Control, US Preventive Services Task Force and } \\
\text { New York state recommendations of one-time screening in patients born between } 1945 \text { and } 1965 . \\
\text { Schedule regular meetings with key members to share successes and opportunities for } \\
\text { improvements and to request allocation of resources to overcome barriers. } \\
\text { - Offer HCV antibody testing to patients born between } 1945 \text { and } 1965 . \\
\text { Increase access for hepatology clinic. }\end{array}$ \\
\hline
\end{tabular}

Patient engagement Physicians review and offer HCV screening in eligible patients.

- Outline patient-related barriers to HCV screening and linkage to care and develop plans to overcome challenges.

- Provide education and engage patients in discussion about chronic HCV infection and complications to improve HCV screening.

- Assign a social worker to improve patient-related barriers of transportation to hepatology clinic.

- Patient navigator assesses barriers of no-shows to hepatology clinic by calling patients.

- Discuss HCV screening in eligible patients at every visit or at least annually for patients who refused in the past.

- Provide education to patients about chronic HCV infection and available treatment to improve understanding and adherence to hepatology clinic appointment.

- Create workflow to ensure that a physician reviews positive HCV RNA results and discusses with patient in a timely manner.

- Offer incentive to patients for linkage to hepatology care.

Leverage health

information

technology
- Design a new electronic health record patient database to identify and track patients for HCV screening.

- Design a new nursing workflow for HCV screening in compliance with NY state 'opt out' consent policy to improve physicians' efficiency during clinic visit.

- Design new HCV nursing workflow in electronic health records for HCV antibody order entry.

- Patient outreach by calling and sending letters who are lost to follow-up in hepatology clinic.

- Track completed HCV antibody test and HCV RNA positive results and ensure physicians' acknowledgement and follow-up.

- Generate registry to track patients who refused HCV screening and allocate resources to overcome barriers.

- Send automated letter to notify patient about negative HCV antibody test result.

Close loops for
referrals and tests

$\mathrm{HCV}$, hepatitis $\mathrm{C}$ virus. 


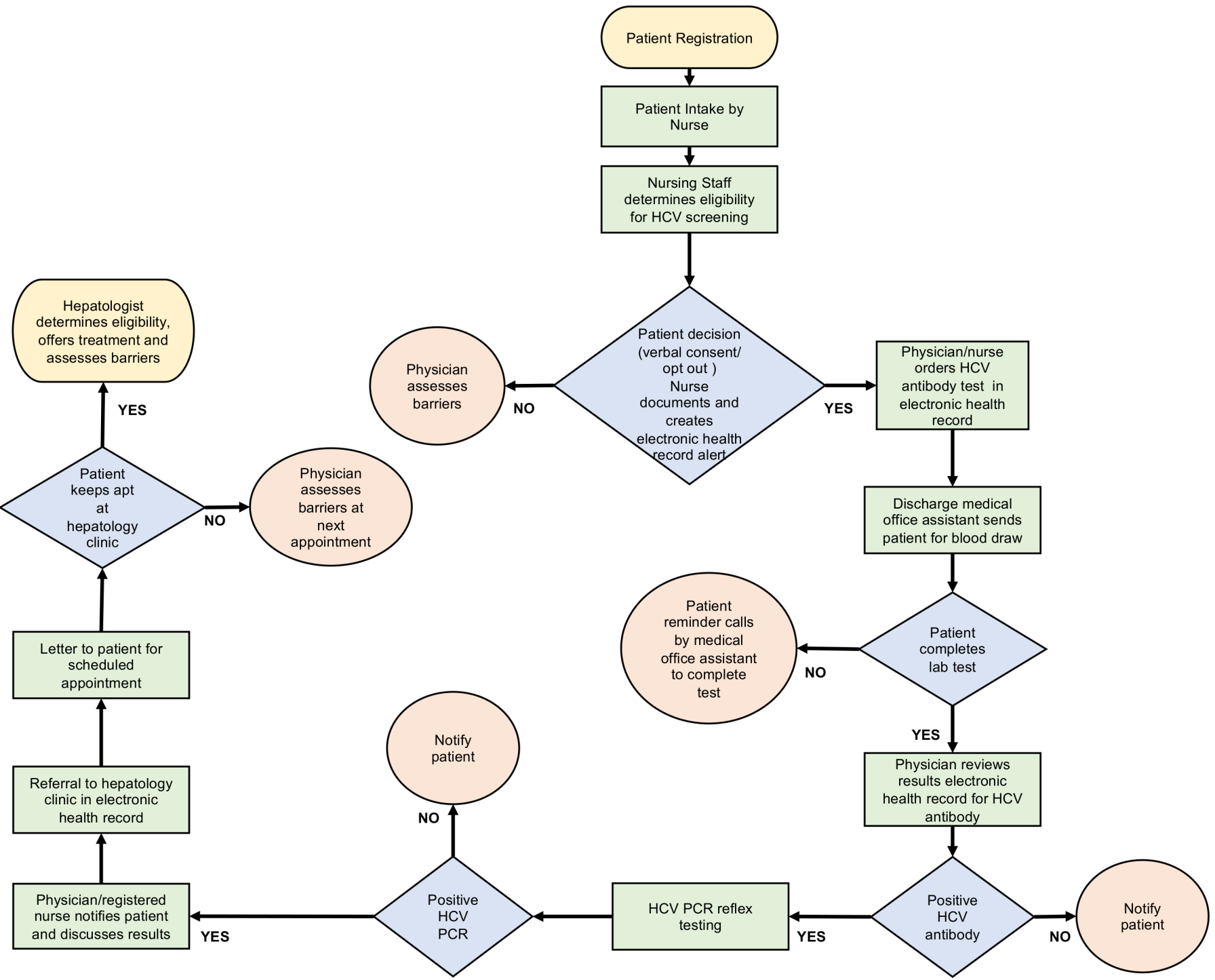

Figure 3 Process flow map for HCV screening in internal medicine clinic. Process workflow chart. HCV, hepatitis C virus.

PDSA cycle 7 (October-December 2016): patient education and nursing reminders

We continued to provide patient education about HCV testing. Nursing staff and physicians reviewed patient education materials with the patients. The physician champion provided refresher training and reminded nursing staff of electronic health record workflow.

\section{PDSA cycle 8 (January-March 2017): redesign of electronic health} record template and nursing workflow

The quality improvement team redesigned the electronic health record template and nursing workflow based on New York State policy changes for HCV screening. The new policy included: (1) elimination of the requirement for verbal consent for HCV screening and changed to an 'opt' out consent policy. Registered nurses inform patients they were ordering HCV testing as a part of routine blood work unless the patient's objected. The nursing staff documented the patient's agreement or disagreement for HCV testing; (2) registered nurses order $\mathrm{HCV}$ antibody tests as a standing order from physicians after patient's agreement; (3) nurses can now create an electronic health record order entry for an HCV antibody test for physician authorisation; and (4) nurses can now notify patients about HCV RNA positive test results and inform the patient about the need for linkage to care to a hepatology clinic. After assessing the patients prior to physician evaluation, the nursing staff created an order entry for HCV antibody test. We also used a patient navigator to facilitate linkage to hepatology care.

\section{PDSA cycle 9 (April-May 2017): electronic health record outage}

On 9 April 2017, the hospital faced an electronic health record outage for 2 months. The quality improvement team leaders continued to provide feedback to the team during this time.

\section{Data analysis}

We analysed data from the electronic patient registry and created a weekly statistical process control chart for process measures of HCV completion rates. 


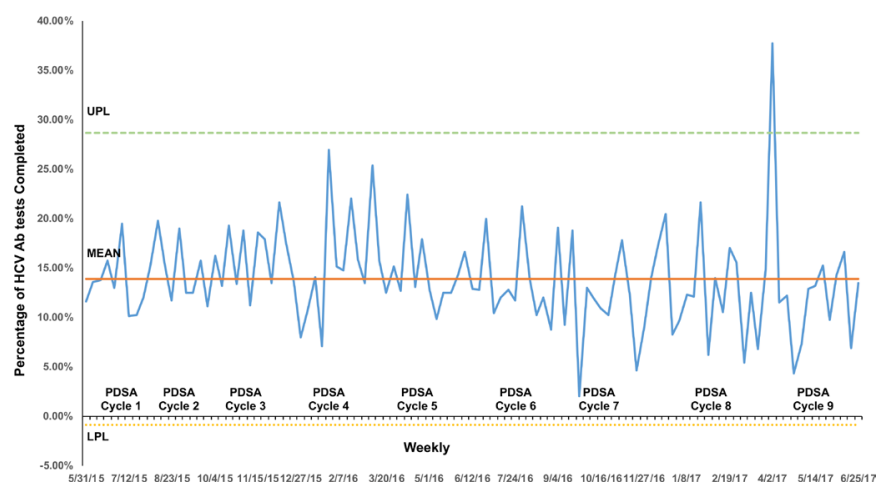

Figure 4 Weekly statistical process control chart (SPC) chart: HCV antibody completion rate. Weekly SPC chart showing percentage of $\mathrm{HCV}$ completion rate in patients born between 1945 and 1965. CL, control limit; HCV, hepatitis C virus; LCL, lower control limit; PDSA, Plan Do Study Act; UCL, upper control limit.

\section{RESULTS}

\section{Demographics}

The mean age of this population was 58.8 and $52.3 \%$ were males. $66.8 \%$ were African-Americans, $28.0 \%$ were white, while $5.1 \%$ were classified as other race. Furthermore, $12.0 \%$ of patients had a history of serious mental illness (schizophrenia or bipolar disorder).

\section{Outcome measures}

HCV antibody screening rate and HCV RNA positive rates

There were total of 1291 individuals born between 1945 and 1965 (unduplicated patients), seen over 24 months; 391 of these were screened for HCV. The average HCV antibody screening rate in eligible patients was $30.3 \%$ (391/1291) during the 24-month study period. Of the 391 patients that were screened, 170 patients tested positive $(43.5 \%)$, and 221 patients tested negative for the HCV antibody. The HCV RNA positive rate was $41.4 \%$ $(162 / 391)$ of the total screened population and $95.3 \%$ $(162 / 170)$ in the HCV antibody positive population. HCV infection was diagnosed in $12.5 \%$ (162/1291) of all eligible patients in the internal medicine clinic and $95.3 \%$ (162/170) in the HCV antibody positive population. We sustained an HCV screening rate of $25.4 \%$ during the postproject 1-year period (May 2017-June 2018).

\section{Linkage to hepatology clinic in patients with confirmed HCV diagnosis}

Seventy percent $(114 / 162)$ of patients with a diagnosis of Chronic HCV were linked to a hepatology clinic within the 24 month project timeframe. Eighty percent (114/162) of patients seen by a hepatologist were treated with direct-acting antivirals, $70 \%(80 / 114)$ of patients achieved sustained viral response and 11 patients have recently started direct-acting antivirals treatment. All patients achieved sustained viral response after completion of HCV treatment. One patient refused treatment, one patient was diagnosed with metastatic liver cancer and other patients are being evaluated for treatment eligibility.

\section{Process measures}

\section{HCV antibody completion rate}

The mean HCV antibody completion rate was $14.0 \%$ in a weekly statistical process chart (figure 4 ). The patterns of weekly HCV order rates and completion rates were identical; therefore, we have displayed only the weekly HCV completion rates. We observed variations during all PDSA cycles, resulting in an unsustainable increase in $\mathrm{HCV}$ completion rate. The highest rate of screening completion, $21.2 \%$, occurred in the week of 31 July 31, 2016. In calculating the weekly HCV completion rate, the denominator was a unique number of patients seen in the clinic in a week. Patients were counted more than once during 24 months if they were eligible for HCV testing during any subsequent clinic visit.

\section{Referrals to a hepatologist in HCV RNA-positive patients}

The majority of patients received a follow-up primary care visit with a physician to discuss their results and provide education on chronic HCV. All patients with confirmed $\mathrm{HCV}$ infection were referred to a hepatology clinic; however, about $50 \%$ of the patients were non-adherent to the first scheduled hepatology clinic appointment. Patents rescheduled after they missed their first apointment and subsequently were treated by a hepatologist. Patients were linked to care and/or treated for HCV within the 24 month project implementation timeframe.

\section{Improvement in physician knowledge}

In the second PDSA cycle, residents answered $60.0 \%$ of pretest questions correctly. Following physician education, residents answered $95 \%$ of questions correctly, indicating an objective improvement in the resident's knowledge.

\section{Balancing measures}

Patient wait time in clinic

There were no reports of an increase in patient complaints or overtime for clinic staff, which suggest that the project may not have adversely impacted patient wait times. Patients spent a total of 1 hour during a follow-up clinic visit including wait and time with the provider.

\section{Cost of the treatment}

Providers, clinic staff and patients identified the cost of HCV treatment as a major barrier due to a higher copay or lack of insurance coverage.

\section{Summary}

We exceeded our goal and achieved 30.3\% HCV screening rate in patients born between 1945 and 1965, from a baseline rate of less than $3 \%$ within 24 months in the internal medicine clinic. The HCV antibody positive rate was $43.5 \%(170 / 391)$, and HCV RNA positive rate was $95.3 \%(162 / 170)$. HCV infection was diagnosed in $12.5 \%(162 / 1291)$ of patients or $41.4 \%(162 / 391)$ of the screened population. Of those positive, $70 \%(114 / 162)$ were linked to hepatology care. Of those screened, $43.5 \%$ tested positive for the HCV antibody, and $95.3 \%$ were 
HCV RNA positive. Seventy percent of HCV RNA-positive patients were linked to care in a hepatology clinic. Eighty percent of those patients seen by a hepatologist were treated with direct-acting antivirals, and $49 \%$ of patients achieved sustained viral response within the study period.

Previous studies have shown that electronic health record embedded alerts markedly increased HCV testing among patients born between 1945 and 1965. ${ }^{23} 27$ 36-39 In this quality improvement project, we implemented multiple changes in the electronic health record nursing workflow. HCV screening was integrated into the routine nursing workflow, and changes to this workflow were subsequently made following an amendment to New York state screening laws. This amendment allowed for a standing order (non-patient specific order) to be made by nurses. These electronic health record workflow changes increased HCV screening rates in patients born between 1945 and 1965 in the internal medicine clinic during the eighth PDSA cycle. However, due to an electronic health record outage, this increase was not sustainable. Educating clinic physicians directly affects HCV screening rates by raising awareness of the disease. ${ }^{40-43}$ We educated the physicians and nursing staff on chronic HCV infection, screening, diagnosis and electronic health record workflow by a PowerPoint presentation with a small group discussion. We observed significant increase in HCV screening rates during the third PDSA cycle of nursing education.

Patients were highly engaged with the team during this project. Major barriers to screening identified by the team were based on physician and nurse's direct discussion with the patients. During the feedback session of the team meetings, various barriers were discussed and some were selected as major barriers due to estimated frequency reported by the patients. Barriers to HCV screening include concerns with cost of testing and treatment. ${ }^{44-49}$ The main barriers for acceptance of HCV screening included lack of awareness of complications of untreated chronic HCV infection, fear of blood drawn, fear of diagnosis and treatment for chronic HCV infection. An additional barrier was the limited amount of time allocated for a patient visit. During the $20-30 \mathrm{~min}$ visit, HCV screening was not the sole focus and the nursing staff were required to perform additional screening tests; therefore, HCV screening was not adequately addressed. Previous studies have shown that barriers to linkage of care include lack of health insurance, incarceration, homelessness and substance abuse. ${ }^{48}$ In the internal medicine clinic, $70 \%$ of patients were linked to care, which is similar to previous studies that have shown various rates from $44 \%$ to $100 \%$ linkage of care..$^{24} 2850$ The main barriers to linkage of care in the internal medicine clinic population included lack of patient knowledge about complications of undiagnosed and untreated HCV, fear of possible adverse effects of treatment, concern about cost of treatment and insurance coverage. The majority of those patients that were lost to follow-up care were diagnosed with serious mental illness (schizophrenia and bipolar disorders).
The hepatology clinic staff was actively engaged in patient outreach to facilitate linkage to care.

A large number of the internal medicine clinic patients born between 1945 and 1965 declined screening for HCV, which is in contrast to previous studies that demonstrate the willingness of this population to be screened $^{22}{ }^{51}$ Furthermore, the HCV prevalence rate in the total internal medicine clinic patients born between 1945 and 1965 was $12.5 \%$ (162/1291), with a prevalence rate of $41.4 \%(162 / 391)$ in those that were screened. This rate is much higher than reported by other studies. For example, an HCV prevalence rate of $8.8 \%$ was reported in the screened birth cohort in an urban primary care clinic, ${ }^{51}$ and an HCV prevalence of $6.7 \%$ in screened individuals born between 1945 and 1965 was reported in a safety net hospital. ${ }^{22}$ The higher HCV prevalence in the internal medicine clinic patients born between 1945 and 1965 may be due to higher prevalence of substance use disorder in this population. In our previous study using this internal medicine clinic population, we found a high prevalence of opioid misuse, $44 \%$ had misuse, $38 \%$ had the appearance of opioid adherence and $17 \%$ had possible opioid nonadherence. ${ }^{52}$ Substance misuse is a well-established barrier to HCV screening. ${ }^{53}$ Furthermore, the internal medicine clinic population consists largely of African-American (66.8\%). HCV has been shown to disproportionately affect African-Americans. ${ }^{4151}$

Fostering open dialogue and exemplifying positive clear communication with multidisciplinary team including patients led to the success of this project. Patients were actively engaged in this quality improvement project in identification of barriers to HCV screening, education and shared decision making for treatment of chronic HCV infection. Previsit planning by the nursing staff to identify eligible patients born between 1945 and 1965 was unsuccessful due to time constraints and limited resources. Due to an increase in demand for hepatology care, the hospital leadership improved access to hepatology clinics. Multiple attempts were made by physicians to review a need for hepatology care in HCV RNA-positive patients; however, physicians identified major barrier of serious mental illness to the acceptance to hepatology care.

Processes and workflows designed during this quality project for improving HCV screening in the internal medicine clinic have become the standard of care. There was an evidence of internal validity; we were able to demonstrate meaningful impact resulting from different PDSA cycles during quality improvement project. We were able to sustain the HCV screening rate; the average HCV screening rate was $25.4 \%$ during the postproject 1-year period (May 2017-June 2018). Future PDSA cycles will include a use of patient navigator to track patient's linkage to hepatology care and improve access to hepatology clinic. Additional PDSA cycles will include interactive workshops for the internal medicine clinic staff and physicians on motivational interviewing technique, pocket cards of HCV screening for the physicians as a 
reminder and patient education with videos and PowerPoint presentation about HCV by use of digital photo frames in the patient examination room. Personalised education to HCV patients may be best achieved through active engagement of nurses and a patient navigator; this may facilitate higher linkage to hepatology care.

\section{Limitations}

This quality improvement project was performed in a safety-net primary care clinic in vulnerable and underserved patient population; therefore, results cannot be generalisable to other settings. This study observed a high rate of chronic infection among patients who were anti-HCV positive. A possible explanation for this finding may be a biased population with higher prevalence rates of substance use disorder. We accomplished $70 \%$ linkage to care in a hepatology clinic, located within the hospital; this result may be different in other settings, with limited access to hepatology in a specific geographic area.

\section{CONCLUSION}

We achieved 30\% HCV screening rates and exceeded our goal in patients born between 1945 and 1965 within 24 months in the internal medicine clinic. HCV screening rate during postproject 1-year period (May 2017-June 2018) was sustainable at $25.4 \%$. Engagement of a multidisciplinary team in performing the root cause analysis and creating driver's diagram was critical to the design of this project. Physician, nursing and patient education was crucial to create awareness of HCV in patients born between 1945 and 1965. The integration of nursing workflow for HCV screening into the normal clinic workflow to promote the normalisation and sustainability of routine testing was the key driver to the success of this quality improvement project. Improving HCV screening and diagnosis has had substantial impact on the quality of care for internal medicine clinic patients at minimal cost. The increase in HCV diagnosis led to treatment and resulted in sustained viral response, leading to prevention of complications from untreated chronic HCV infection. This may be translated to a reduction in the burden of cost to patients and third-party payers. The interventions implemented in this quality improvement can be replicated in other settings to improve the quality of care delivery.

Acknowledgements We would like to thank the internal medicine and hepatology clinic staff and the information technology department at the hospital.

Contributors SB: study oversight, study concept and design, acquisition of data, analysis and interpretation of data, drafting of manuscript, critical revision of the manuscript for important intellectual content and finalisation of manuscript ME-A, NN and TA: acquisition of data, analysis and interpretation of data. JLR: interpretation of data, drafting of manuscript, critical revision of the manuscript for important intellectual content and finalisation of manuscript.

Funding Research reported in this publication was supported by the National Center for Advancing Translational Sciences of the National Institutes of Health under award Number UL1TR001412 and Al129649.

Disclaimer The content is solely the responsibility of the authors and does not necessarily represent the official views of the National Institutes of Health.
Competing interests None declared.

Patient consent for publication Not required.

Provenance and peer review Not commissioned; externally peer reviewed.

Open access This is an open access article distributed in accordance with the Creative Commons Attribution Non Commercial (CC BY-NC 4.0) license, which permits others to distribute, remix, adapt, build upon this work non-commercially, and license their derivative works on different terms, provided the original work is properly cited, appropriate credit is given, any changes made indicated, and the use is non-commercial. See: http://creativecommons.org/licenses/by-nc/4.0/.

\section{REFERENCES}

1 Chak E, Talal AH, Sherman KE, et al. Hepatitis C virus infection in USA: an estimate of true prevalence. Liver International 2011;31:1090-101.

2 Saab S, Le L, Saggi S, et al. Towards the elimination of hepatitis C in the United States. Hepatology 2017.

3 CDC. Overview and statistics. Available: https://www.cdc.gov/ hepatitis/hcv/hcvfaq.htm\#section1.

4 Edlin BR, Eckhardt BJ, Shu MA, et al. Toward a more accurate estimate of the prevalence of hepatitis $C$ in the United States. Hepatology 2015;62:1353-63.

5 Younossi ZM, Stepanova M, Afendy M, et al. Knowledge about infection is the only predictor of treatment in patients with chronic hepatitis C. J Viral Hepat 2013;20:550-5.

6 Denniston MM, Klevens RM, McQuillan GM, et al. Awareness of infection, knowledge of hepatitis $\mathrm{C}$, and medical followup among individuals testing positive for hepatitis C: National health and nutrition examination survey 2001-2008. Hepatology 2012;55:1652-61.

7 Bragg DA, Crowl A, Manlove E. Hepatitis C: a new era. Prim Care 2017;44:631-42.

8 Mah'moud MA. Current management of hepatitis $C$ virus infection. $N$ C Med J 2016;77:188-93.

9 Tamborini Permunian E, Gervaso L, Gerdes V, et al. Direct-Acting antiviral drugs for chronic hepatitis $\mathrm{C}$ and risk of major vascular events: a systematic review. Intern Emerg Med 2018;13:775-90.

10 Konjeti VR, John BV. Interaction between hepatocellular carcinoma and hepatitis $\mathrm{C}$ eradication with direct-acting antiviral therapy. Curr Treat Options Gastroenterol 2018;16:203-14.

11 Axley P, Ahmed Z, Ravi S, et al. Hepatitis C virus and hepatocellular carcinoma: a narrative review. J Clin Trans/ Hepatol 2018;6:1-6.

12 Razavi H, ElKhoury AC, Elbasha E, et al. Chronic hepatitis C virus (HCV) disease burden and cost in the United States. Hepatology 2013;57:2164-70.

13 Tandon N, Reddy KR, Balart LA, et al. Direct and indirect cost burden of chronic hepatitis C. Am J Pharm Benefits 2015;7:e90-100.

14 Denniston MM, Jiles RB, Drobeniuc J, et al. Chronic hepatitis C virus infection in the United States, National health and nutrition examination survey 2003 to 2010. Ann Intern Med 2014;160:293300-300.

15 CDC. Hepatitis C, why people born from 1945-1965 should get tested, 2016. Available: https://www.cdc.gov/knowmorehepatitis/ media/pdfs/factsheet-boomers.pdf

16 Jemal A, Fedewa SA. Recent hepatitis $\mathrm{C}$ virus testing patterns among baby boomers. Am J Prev Med 2017;53:e31-3.

17 Moyer VA. Screening for hepatitis $C$ virus infection in adults: U.S. preventive services Task force recommendation statement. Ann Intern Med 2013;159:349-57.

18 Smith BD, Morgan RL, Beckett GA, et al. Hepatitis C virus testing of persons born during 1945-1965: recommendations from the centers for disease control and prevention. Ann Intern Med 2012;157:817-22.

19 Barocas JA, Wang J, White LF, et al. Hepatitis C testing increased among baby boomers following the 2012 change to CDC testing recommendations. Health Aff 2017;36:2142-50.

20 Kasting ML, Giuliano AR, Reich RR, et al. Hepatitis C virus screening trends: serial cross-sectional analysis of the National health interview survey population, 2013-2015. Cancer Epidemiol Biomarkers Prev 2018;27:503-13.

21 Cdc urges universal hepatitis $\mathrm{C}$ screening for baby boomers. Harvard women's health watch 2012;19:1.

22 Turner BJ, Taylor BS, Hanson J, et al. High priority for hepatitis $\mathrm{C}$ screening in safety net hospitals: results from a prospective cohort of 4582 hospitalized baby boomers. Hepatology 2015;62:1388-95.10.1002/hep.28018

23 Sidlow R, Msaouel P. Improving hepatitis C virus screening rates in primary care: a targeted intervention using the electronic health record. J Healthc Qual 2015;37:319-23. 
24 Goel A, Sanchez J, Paulino L, et al. A systematic model improves hepatitis $\mathrm{C}$ virus birth cohort screening in hospital-based primary care. J Viral Hepat 2017;24:477-85.

25 Roberts K, Macleod J, Metcalfe C, et al. Hepatitis C - Assessment to Treatment Trial (HepCATT) in primary care: study protocol for a cluster randomised controlled trial. Trials 2016;17:366.

26 HRSA. Hepatitis and primary care integration. Available: https://bphc. hrsa.gov/qualityimprovement/clinicalquality/hepatitis/10/25/2018

27 Konerman MA, Thomson M, Gray K, et al. Impact of an electronic health record alert in primary care on increasing hepatitis $\mathrm{C}$ screening and curative treatment for baby boomers. Hepatology 2017;66:1805-13.

28 Trinh JandTurner N. Improving adherence to hepatitis $\mathrm{C}$ screening guidelines. BMJ Open Qual 2018;7.

29 New York state department of health hepatitis C testing law, 2014. Available: https://www.health.ny.gov/publications/1820.pdf

30 Flanigan CA. Implementing the Nation's First State Hepatitis C Testing Law, 2015. Available: https://www.hiv.gov/blog/ implementing-the-nations-first-state-hepatitis-c-testing-law

31 Falade-Nwulia O, Suarez-Cuervo C, Nelson DR, et al. Oral directacting agent therapy for hepatitis $\mathrm{C}$ virus infection. Ann Intern Med 2017;166:637-48.

32 Falade-Nwulia O, Sulkowski MS, Merkow A, et al. Understanding and addressing hepatitis $\mathrm{C}$ reinfection in the oral direct-acting antiviral era. J Viral Hepat 2018;25:220-7.

33 Instiute for healthcare improvement. science of improvement: establishing measures. Available: http://www.ihi.org/resources/ Pages/Howtolmprove/ScienceoflmprovementEstablishingMeasures. aspx

34 Quality AfHRa. Health literacy universal precautions toolkit, 2nd edition, Plan-Do-Study-Act (PDSA) directions and examples, 2015. Available: https://www.ahrq.gov/professionals/quality-patient-safety/ quality-resources/tools/literacy-toolkit/healthlittoolkit2-tool2b.html

35 Stausmire JM, Ulrich C. Using Squire 2.0 as a guide for planning your quality improvement project. Crit Care Nurse 2017;37:72-80.

36 Federman AD, Kil N, Kannry J, et al. An electronic health Recordbased intervention to promote hepatitis $\mathrm{C}$ virus testing among adults born between 1945 and 1965: a cluster-randomized trial. Med Care 2017:55:590-7.

37 Golden MR, Duchin J, Chew LD, et al. Impact of an electronic medical Record-Based system to promote human immunodeficiency virus/hepatitis $C$ virus screening in public hospital primary care clinics. Open Forum Infectious Diseases 2017;4

38 Al-hihi E, Shankweiler C, Stricklen D, et al. Electronic medical record alert improves HCV testing for baby boomers in primary care setting: adults born during 1945-1965. BMJ Open Qual 2017;6:e000084.

39 MacLean CD, Berger C, Cangiano ML, et al. Impact of electronic reminder systems on hepatitis $\mathrm{C}$ screening in primary care. J Viral Hepat 2018;25:939-44.
40 Naghdi R, Seto K, Klassen C, et al. A hepatitis C educational needs assessment of Canadian healthcare providers. Canadian Journal of Gastroenterology and Hepatology 2017;2017:1-10.

41 Miller LS, Rollin F, Fluker S-A, et al. High-Yield birth-cohort hepatitis $C$ virus screening and linkage to care among underserved African Americans, Atlanta, Georgia, 2012-2013. Public Health Rep 2016;131(2 suppl):84-90.

42 Madhani K, Aamar A, Chia D. Hepatitis C screening: the downstream dissemination of evolving guidelines in a resident continuity clinic. Cureus 2017;9:e1441.

43 Samuel ST, Martinez AD, Chen Y, et al. Hepatitis C virus knowledge improves hepatitis $\mathrm{C}$ virus screening practices among primary care physicians. World J Hepatol 2018;10:319-28.

44 Barocas JA, Brennan MB, Hull SJ, et al. Barriers and facilitators of hepatitis $\mathrm{C}$ screening among people who inject drugs: a multi-city, mixed-methods study. Harm Reduct J 2014;11:1.

45 Howes N, Lattimore S, Irving WL, et al. Clinical care pathways for patients with hepatitis $\mathrm{C}$ : reducing critical barriers to effective treatment. Open Forum Infectious Diseases 2016;3.

46 Overbeck K, Bruggmann P, Helbling B. Chronic hepatitis C virus infection in Swiss primary care practices: low case loads-high barriers to treatment? European Journal of General Practice 2011;17:103-8.

47 Rogal SS, McCarthy R, Reid A, et al. Primary care and hepatology Provider-Perceived barriers to and facilitators of hepatitis $\mathrm{C}$ treatment Candidacy and adherence. Dig Dis Sci 2017;62:1933-43.

48 Taylor BS, Hanson JT, Veerapaneni P, et al. Hospital-Based hepatitis C screening of baby boomers in a majority Hispanic South Texas cohort: successes and barriers to implementation. Public Health Rep 2016;131(2_suppl):74-83.

49 Wansom T, Falade-Nwulia O, Sutcliffe CG, et al. Barriers to hepatitis $\mathrm{C}$ virus $(\mathrm{HCV})$ treatment initiation in patients with human immunodeficiency Virus/HCV coinfection: lessons from the interferon era. Open Forum Infect Dis 2017;4.

50 Younossi ZM, LaLuna LL, Santoro JJ, et al. Implementation of baby Boomer hepatitis $C$ screening and linking to care in gastroenterology practices: a multi-center pilot study. BMC Gastroenterol 2016;16:45

51 Geboy AG, Mahajan S, Daly AP, et al. High hepatitis C infection rate among baby boomers in an urban primary care clinic: results from the HepTLC initiative. Public Health Rep 2016;131(2_suppl):49-56.

52 Bakhai, MD, MPH, FACP S, Thilagar, MD B, Reynolds, PhD JL, et al. Correlates of opiate misuse based on aberrant urine drug tests for patients on chronic opiate therapy in a safety-net, academic primary care clinic. J Opioid Manag 2018;14:23-33.

53 Crowley D, Cullen W, Laird E, et al. Exploring patient characteristics and barriers to hepatitis $\mathrm{C}$ treatment in patients on opioid substitution treatment attending a community based fibro-scanning clinic. J Transl Int Med 2017;5:112-9. 